\title{
On the selection of fractional-differential model of convective diffusion with mass exchange
}

https://doi.org/10.31713/MCIT.2020.02

\author{
Vsevolod Bohaienko \\ V.M. Glushkov Institute of Cybernetics of NAS of Ukraine \\ Kyiv, Ukraine \\ sevab@ukr.net
}

\begin{abstract}
The paper considers two fractionaldifferential models of convective diffusion with mass exchange and proposes a decision-making algorithm for determining the optimal model at the time of concentration field observation. As for soils of fractal structure, direct experimental determination of model parameters' values and type of mass exchange process is in many cases impossible, calibration and determination of the most adequate models is performed mainly solving inverse problems by, in particular, meta-heuristic algorithms that are computationally complex. In order to reduce the computational complexity, we study the qualitative differences between diffusion processes described by fractional-differential models with non-local mass exchange on the base of the Caputo derivative and local non-linear mass exchange based on the nonequilibrium sorption equation that corresponds to the description by the Caputo-Fabrizio derivative. We determine under which conditions both models within a given accuracy describe the same set of measurements at a certain moment of time. When the solutions are close at a certain initial stage of process development, the model with the Caputo derivative describes its faster approach to a steady state. Based on the obtained estimates of differences in solutions, a decision-making algorithm is proposed to determine the most accurate model and the values of its parameters concurrently with the acquisition of measurements. This algorithm's usage reduces the time spent on solving inverse calibration problems.
\end{abstract}

Keywords - convective diffusion, mass exchange, fractional-differential models, decision-making algorithm

\section{INTRODUCTION}

The paper deals with certain issues arising while calibrating models of convective diffusion with mass exchange in soils of fractal structure. Such models [1, 2] describe diffusion processes with the transition from normal diffusion to subdiffusion and subsequent return to normal diffusion mode [2].

Since the exact determination of parameters' values for fractional-differential models is currently an open problem,

\author{
Anatolij Gladky \\ V.M. Glushkov Institute of Cybernetics of NAS of Ukraine \\ Kyiv, Ukraine \\ gladky@ukr.net
}

selecting (fitting) them in a way to make the model best describe the available measurements remains in many cases the only approach when applying them in practice. Such fitting can be performed particularly using meta-heuristic algorithms that are computationally complex. As the type of mass transfer processes is also usually unknown, the problem of choosing the most accurate model further increases the complexity of accurate forecasting of diffusion processes, which include, e.g., pollution propagation that should be predicted promptly in the case of accidents. In such a situation, it is important to develop approximate decision-making algorithms that will allow choosing the model that is the most accurate for the observed process.

As it was shown by the results of the comparisons given in [2], with the best chosen parameter values, the solutions by different fractional-differential models of diffusion processes that are close at the initial stages of process development further become significantly different. So it is relevant to determine the qualitative differences in the anomalous diffusion processes described by different models, solutions of which initially differ from each other not more than by the level of measurement errors. The mathematical description of these differences allows determining the most accurate model in the process of observing changes in pollutant concentration fields.

\section{INITIAL-BOUNDARY VALUE PROBLEMS AND NUMERICAL METHOD}

The two-dimensional fractional-differential equation of convective diffusion of soluble substances with mass exchange can be stated as [3]

$$
\sigma D_{t}^{(\beta)} C+\frac{\partial N}{\partial t}=L C, L=D \Delta_{x y} C-v_{x} \frac{\partial C}{\partial x}-v_{y} \frac{\partial C}{\partial y}
$$

where $C$ is the concentration of the substance in liquid (mobile) phase, $N$ is its concentration in solid (immobile) phase, $\sigma$ is the porosity, $v_{x}=v_{x}(x, y), v_{y}=v_{y}(x, y)$ are the components of filtration velocity vector, $D=$ const is the convective diffusion coefficient, $\Delta_{x y}$ is the Laplace operator with respect to the variables $x, y$, 


\section{Modeling, control and information technologies $-\mathbf{2 0 2 0}$}

$D_{t}^{(\beta)} p(t)=\frac{1}{\Gamma(1-\beta)} \int_{0}^{t} \frac{p^{\prime}(\tau) d \tau}{(t-\tau)^{\beta}}$ is the Caputo derivative of the order $\beta(0<\beta<1), \Gamma(z)$ is the gamma function.

We consider two options for modelling the dynamics of particle outflow into the immobile phase. According to [4] it can be described as

$$
\frac{\partial N}{\partial t}=\bar{\beta} D_{t}^{(\gamma)} C, 0<\gamma<1
$$

where $\bar{\beta}$ is the mass exchange rate parameter.

The second considered model is obtained by adding to the equation (1) the non-equilibrium sorption equation $[5,6]$

$$
\frac{\partial N}{\partial t}=\beta_{*}\left(\sigma C-\alpha_{*} N\right)
$$

where $\alpha_{*}=1 / \Gamma, \Gamma$ is the Henry's coefficient [7], $\beta_{*}$ is the mass exchange rate parameter.

Similarly to [4], solving (3) with respect to $N$ with the initial conditions $\left.C\right|_{t=0}=\left.N\right|_{t=0}=0$, we obtain

$$
\begin{gathered}
N(t)=\beta_{*} \sigma \int_{0}^{t} C_{3}(t-\tau) e^{-\alpha_{*} \beta_{*} \tau} d \tau, \\
\frac{\partial N}{\partial t}=\beta_{*} \sigma \int_{0}^{t} C_{3}^{\prime}(\tau) e^{-\alpha_{*} \beta_{*}(t-\tau)} d \tau= \\
=\beta_{*} \sigma(1-\chi)^{C F C} D_{t}^{\chi} C, \chi=\frac{\alpha_{*} \beta_{*}}{1+\alpha_{*} \beta_{*}},
\end{gathered}
$$

where ${ }^{C F C} D_{t}^{\chi} C$ is the fractional Caputo-Fabrizio derivative [8] or the order $\chi$.

Thus, it can be noted that the main difference between the mass exchange equations (2) and (3) is the form of integral operator's kernel - power in (2) and exponential in (3).

To simplify the description, we consider the filtration problem that has an analytical solution. Let the mass transfer of contaminants from a reservoir occurs in the domain $G_{z}$ given in [9], Fig. 1a. For such a scheme, we can perform a transition into the domain of complex flow potential $G_{\omega}, \omega=\varphi+i \psi(\psi$ is the flow function), which has the form of a horizontal halfband ([9], Fig. 1b), using a conformal mapping method. The closed-form solution of the corresponding filtration problem in this domain is well-known.

Setting the concentration $C_{0}$ of solute at the inlet $A C$ of the filtration stream, the boundary and initial conditions for the models (1), (2), and (1), (3) can be written in the form

$$
\left.C\right|_{A C}=C_{0},\left.\frac{\partial C}{\partial n}\right|_{A B, C B}=0,
$$

$$
\left.C\right|_{t=0}=\left.N\right|_{t=0}=0
$$

where $n$ is the outer normal to the corresponding curve, $A B$ is the flow symmetry axis, $C B$ is the flow line ([9], Fig.1a).

Numerical solution of initial-boundary value problems for the models (1), (2) and (1), (3) is performed according to the locally one-dimensional schemes [10] given in [11] and [12], respectively. We use a uniform finite-difference grid, which covers the domain of complex flow potential restricted from the right by the line $\varphi=\varphi_{0} \quad\left(\varphi_{0}>>1\right)$; first-order finitedifference analogue of the fractional differentiation operator; the Thomas algorithm [10] for solving three-diagonal linear equations systems obtained after discretization.

The problem of theoretical analysis of the convergence and stability of the used schemes is beyond the scope of this paper. For schemes of this class, they are considered for the diffusion equation, particularly, in [13].

\section{ESTIMATES OF DIFFERENCES BETWEEN PROBLEMS’ SOLUTIONS}

The models (1), (2) and (1), (3) describe diffusion processes with different mechanisms of mass exchange - nonlocal in time but linear in the case of equation (2) and local but non-linear in the case of equation (3). When modelling real processes, it is necessary to calibrate the models by determining the values of their parameters based on available measurements that can be inaccurate. Given that the mechanism of mass exchange in the particular case is often unknown, identifying the model that best describes the observed process is also urgent. Hence, we study the conditions under which both models, within a given accuracy, describe the same set of measurements obtained at a specific point in time, that is, when the solutions obtained by the models (1), (2) and (1), (3) differ by no more than a given value.

We construct the estimates of the maximal difference between the solutions upon the models (1), (2), and (1), (3) and the solution $C_{1}$ of the diffusion equation (1) without taking mass exchange into account.

Let $C_{2}=C_{1}+\omega_{1}$ satisfies the equations (1), (2). Then, subtracting from the equation (1) for $C_{2}$ the corresponding equation without taking mass exchange into account for $C_{1}$, we obtain

$$
\bar{\beta} D_{t}^{(\gamma)} C_{2}=\left(L-\sigma D_{t}^{(\beta)}\right) \omega_{1} .
$$

Assuming that $\quad\left|\left(L-\sigma D_{t}^{(\beta)}\right) \omega_{1}\right| \geq M_{1} \max \left|\omega_{1}\right|$, $\partial C_{2} / \partial t \leq M_{2}$ and, correspondingly, $D_{t}^{(\gamma)} C_{2} \leq M_{2} \frac{t^{1-\gamma}}{\Gamma(2-\gamma)}$, the upper estimate of the maximal difference has the form

$$
\max \left|\omega_{1}\right| \leq \bar{\omega}_{1}=M_{3} K_{1}, K_{1}=\frac{\bar{\beta} t^{1-\gamma}}{\Gamma(2-\gamma)}, M_{3}=M_{2} / M_{1} .
$$




\section{Modeling, control and information technologies - 2020}

For the solution $C_{3}=C_{1}+\omega_{2}$ of the model (1),(3) we have

$$
\frac{\partial N}{\partial t}=\left(L-\sigma D_{t}^{(\beta)}\right) \omega_{2}
$$

Assuming that $\quad\left|\left(L-\sigma D_{t}^{(\beta)}\right) \omega_{2}\right| \geq M_{1} \max \left|\omega_{2}\right| \quad$ and $\partial C_{3} / \partial t \leq M_{2}$, the upper estimate of the maximal difference has the form

$$
\max \left|\omega_{2}\right| \leq \bar{\omega}_{2} \approx M_{3} K_{2}, K_{2}=\frac{\sigma}{\alpha_{*}}\left(1-e^{-\alpha_{*} \beta_{*} t}\right) .
$$

Since the propagation of the concentration front is delayed when mass exchange is considered comparing to the situation of modelling diffusion without taking it into account [11, 12], the maximal difference between the solutions of (1), (2) and (1), (3) can be estimated as $d\left(C_{2}, C_{3}\right) \approx\left|\bar{\omega}_{2}-\bar{\omega}_{1}\right|$.

\section{ALGORITHM FOR DECISION-MAKING IN THE PROCESS OF FORECAST}

When it is necessary to forecast the observed anomalous diffusion process in a situation when the actual mass exchange mechanism is unknown and, accordingly, it is impossible to a priori determine the most appropriate mathematical model, we propose the following decision-making algorithm:

- Assume that we have the measurements $C$ of the concentration in the moments of time $\tau_{i}, i=1, \ldots, n$. Perform a calibration of the model (1), (2), that is, select, e.g. using some meta-heuristic optimization algorithm, the values of the parameters under which it best describes the available measurements;

- Carry out simulations with the defined parameter values and with $\bar{\beta}=0$. Obtain the values of maximal absolute differences $\max \left|\omega_{1}\left(\tau_{i}\right)\right|, i=1, \ldots, n$. Use the estimate (4) to find the value of $M_{3}$;

- Solve the system of non-linear equations $M_{3} K_{2}\left(\tau_{i}\right)=\max \left|\omega_{1}\left(\tau_{i}\right)\right|, i=1, \ldots, n$, e.g. by some gradient method, and obtain the values of the parameters of the model (1), (3) that, according to the estimate (5), allow describing the process close to the observed one;

- $\quad$ Perform predictive modelling by (1), (2) and (1), (3) for $t>\tau_{n}$;

- When receiving the next series of measurements, compare them with the results of the predictive modelling and select the model that best describes them for further forecast.

The usage of this algorithm makes it possible to determine the mathematical model that is more relevant to the observed process, reducing the time spent on solving inverse problems for calibration as it is not needed to be performed for all considered models.

\section{EXPERIMENTAL COMPARISON OF THE SOLUTIONS}

Performing computational experiments, we determine the values of the parameters $\beta^{*}$ and $\alpha^{*}$ of the model (1), (3) and estimate the constant $M_{3}$ according to the above-described algorithm for the fixed parameters $\bar{\beta}$ and $\gamma$ of the model (1), (2) using solutions at certain moments of time and study how in such a situation the difference between solutions behave at other moments.

We got the base solutions for different $\bar{\beta}, \gamma$ at $\beta=0.8$, $\sigma=0.8, L=22, H=1 / \sqrt{5}, \bar{k}=2 \sqrt{5} /(2.5 \pi), \varphi_{0}=5, m=20$, $n=30$, grid size of $20 \times 30$ cells, and $\tau=0.0001$ for $t=0.001,0.002, \ldots, 0.01$. The calculated values of $\beta^{*}$ and $\alpha^{*}$ that allow obtaining solutions close to the solutions by the model (1), (2), are given in Table 1.

TABLE I. VALUES OF MODELS' COEFFICIENTS

\begin{tabular}{|l|l|l|l|l|}
\hline $\bar{\beta}$ & $\gamma$ & $\alpha^{*}$ & $\beta^{*}$ & $M_{3}$ \\
\hline 0.2 & 0.6 & 23,03 & 30 & 1,35 \\
\hline 0.6 & 0.6 & 7,24 & 103 & 1,22 \\
\hline 0.2 & 1.0 & 3,75 & 449 & 0,96 \\
\hline 0.7 & 0.8 & 2,76 & 606 & 0,95 \\
\hline 0.6 & 1.0 & 1,3 & 2876 & 0,72 \\
\hline
\end{tabular}

Considering the dependencies of the models' coefficients on the maximum absolute differences ("delays") from the solution of the problem without taking mass exchange into account, let us note that for the model (1), (3), the necessary value of $\beta^{*}$ increases exponentially with the increase of delay and $\alpha^{*}$ decreases with the order $x^{-1.28}$. The coefficient of determination for both dependencies here is greater than $R^{2}=0.94$. The parameters of the model (1), (2) with the Caputo derivative are not so clearly correlated with the maximal absolute difference from the solution without taking mass exchange into account, but for both of them the dependencies are close to linear. So, regarding the convergence of algorithms for solving inverse problems of parameters' values identification, it is generally more convenient to use the model (1), (2) because the sensitivity of its parameters to changes in input data is close to linear.

The maximal differences $\bar{\omega}_{1}, \bar{\omega}_{2}$ between the solutions by the models (1), (2) and (1), (3) and the solution by the model that does not take mass exchange into account along with their estimates according to (4), (5) at different moments of time are depicted for the case of $\bar{\beta}=0.7, \gamma=0.8 \quad\left(\beta_{*}=606\right.$, $\alpha_{*}=2.764$ ) in Fig. 2. The changes in time of the difference between the solutions by the models (1), (2) and (1), (3) for different parameters' values and their estimates according to (4), (5) are shown in Fig. 3. 


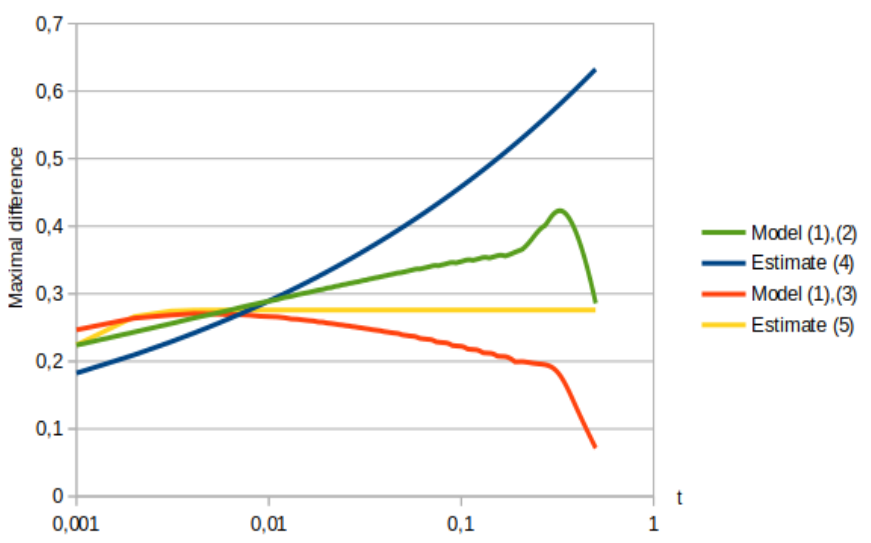

Fig. 1. Maximal absolute differences comparing with the solution by the model without taking mass exchange into account and their estimates for

$$
\bar{\beta}=0.7, \gamma=0.8\left(\beta_{*}=606, \alpha_{*}=2.764\right)
$$

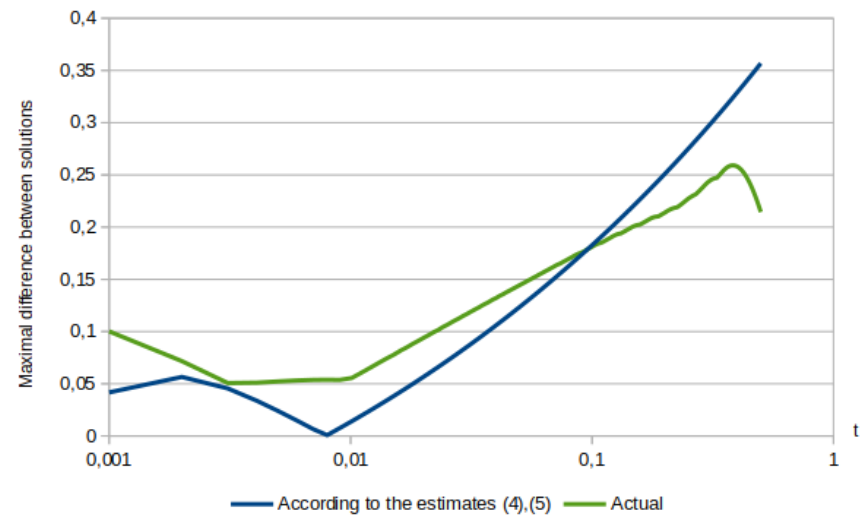

Fig. 2. Differences between the solutions upon the models (1),(2) and

$$
\text { (1), (3) for } \bar{\beta}=0.7, \gamma=0.8\left(\beta_{*}=606, \alpha_{*}=2.764\right)
$$

In general, adequately describing the tendency of changes in the differences between the solutions obtained by the considered models, estimates (4), (5) show that at $\gamma \neq 1$ and $t>0.01$ the model (1), (2) describes the process of concentration field's convergence to a steady state faster than the model (1), (3). When $\gamma=1$, the differences between the solutions are, as expected, small.

Talking about the speed of computations, we can note that in the conducted experiments, comparing with the simulation without taking mass exchange into account, the time spent on the simulation at $t=0.01$ for the model (1), (3) was greater by $\sim 6 \%$. In the case of the model (1), (2), it was greater by $\sim 12 \%$ for $\gamma=1$ and by $\sim 31 \%$ for $\gamma \neq 1$. The convergence rate of the finite-difference scheme for the model (1), (3) was higher when $\tau$ decreases comparing to the model (1), (2).

\section{CONCLUSIONS}

Summarizing we can draw the following conclusions:
- The models (1), (3), and (1), (2) allow describing anomalous diffusion processes that, within a given measurement accuracy, coincide at a certain time interval. With the further development of the process, the model (1), (2) with the Caputo derivative describes its faster convergence to a steady state when $\gamma \neq 1$;

- The dependencies of the model's (1), (2) parameters on the delay when comparing to the solution by the model without taking mass exchange into account are more uniform than for the model (1), (3), making the model (1), (2) with the Caputo derivative a better choice for the algorithms for solving inverse parameters' identification problems;

- The computation speed of the finite-difference scheme for the model (1), (3) is higher than for the case of the model (1), (2) due to the lower computational complexity and better convergence rate.

\section{REFERENCES}

[1] M.M. Meerschaert, Y. Zhang, and B. Baeumer, "Tempered anomalous diffusion in heterogeneous systems," Geophys. Res. Lett., vol. 35(17), L17403, 2008.

[2] H.G. Sun, Zh. Li, Y. Zhang, and W. Chena, "Fractional and fractal derivative models for transient anomalous diffusion: Model comparison," Chaos, Solitons and Fractals, vol. 102, pp. 346-353, 2017.

[3] M.Th. van Genuchten and P.J. Wierenga, "Mass transfer studies in sorbing porous media, 1: Analytical solutions," Soil Science Society of America Journal, vol. 40, pp. 473-480, 1976.

[4] R. Shumer, D.A. Benson, M.M. Meershaert, and B. Baeumer, "Fractal mobile/immobile solute transport," Water Resour. Res., vol. 39(10), pp. 1296-1309, 2003.

[5] I.I. Liashko, L.I. Demchenko, and G.E. Mystetsky, "Numerical solution of the problems of heat and mass transfer in porous media (in Russian)," Kyiv: Naukova Dumka, 1991.

[6] P. Ia. Polubarinova-Kochina, "Groundwater movement theory (in Russian)," Moscow: Nauka, 1977.

[7] N.N. Verigin, S.V. Vasiliev, and N.R. Kuranov "Method for forecasting of salt regime of soil and groundwater (in Russian)," Moscow: Kolos, 1979.

[8] M. Caputo and M. Fabrizio, "A new definition of fractional derivative without singlular kernel,” Prog. Fract. Differ. Appl., vol. 1(2), pp. 73-85, 2015.

[9] V.M. Bulavatsky, "Mathematical modeling of dynamics of the process of filtration convection diffusion under the condition of time nonlocality," Journal of Automation and Information Science, vol. 44(2), pp.13-22, 2012.

[10] A. Samarskii, "The Theory of Difference Schemes," New York: CRC Press, 2001.

[11] V.M. Bulavatsky and V.O. Bohaienko, "Some boundary value problems of fractional-differential mobile-immobile migration dynamics in a profile filtration flow," Cybernetics and Systems Analysis, in press.

[12] V.A. Bohaienko and V.M. Bulavatskiy, "Computer Simulation Based on Non-local Model of the Dynamics of Convective Diffusion of Soluble Substances in the Underground Filtration Flow under Mass Exchange Conditions," Journal of Automation and Information Science, vol. 51(5), pp.16-29, 2019.

[13] A. Chen and C.P. Li, "A novel compact ADI scheme for the timefractional subdiffusion equation in two space dimensions," Int. J. Comput. Math., vol. 93(6), pp. 889-914, 2016. 